\title{
Descolamento reg'matogênico de retina: avaliação pós-operatória da mácula
}

\author{
Rhegmatogenous retinal detachment: a postoperative study of the macula
}

\author{
Otacílio de Oliveira Maia Júnior ${ }^{1}$ \\ Walter Yukihiko Takahashi ${ }^{2}$ \\ Maria Teresa Brizzi Chizzotti Bonanomi ${ }^{3}$ \\ Tiago Eug'ênio Faria e Arantes ${ }^{4}$
}

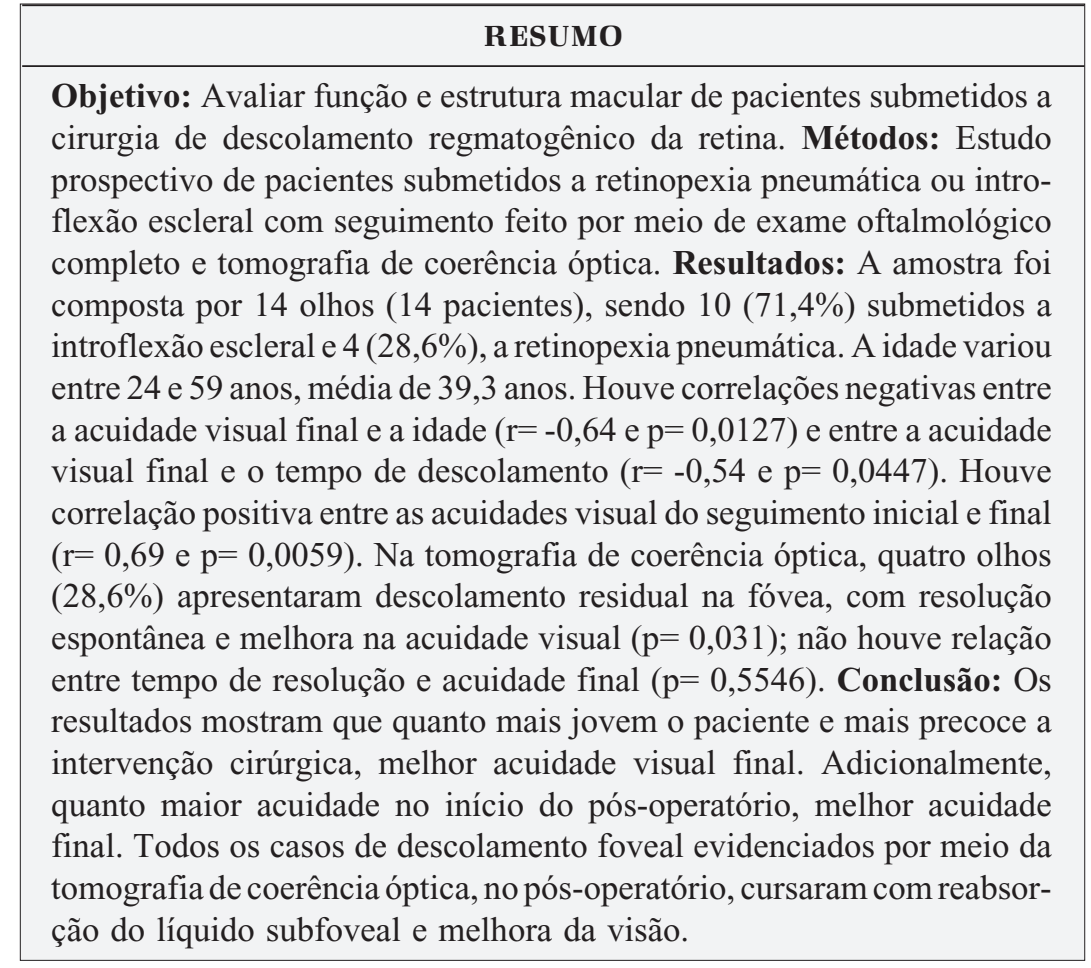

Trabalho desenvolvido no Departamento de Oftalmologia do Hospital das Clínicas da Faculdade de Medicina da Universidade de São Paulo - USP - São Paulo (SP) - Brasil.

${ }^{1}$ Pós-Graduando (Nível Doutorado) da Faculdade de Medicina da Universidade de São Paulo - USP - São Paulo (SP) - Brasil. Especialista em Retina e Vítreo do Departamento de Oftalmologia do Hospital das Clínicas da Faculdade de Medicina da Universidade de São Paulo USP - São Paulo (SP) - Brasil.

${ }^{2}$ Médico Assistente Doutor e Chefe do Setor de Retina e Vítreo do Departamento de Oftalmologia do Hospital das Clínicas da Faculdade de Medicina da USP - São Paulo (SP) - Brasil.

${ }^{3}$ Médica Assistente Doutora do Departamento de Oftalmologia do Hospital das Clínicas da Faculdade de Medicina da USP - São Paulo (SP) - Brasil.

${ }^{4}$ Médico Estagiário de $2^{\circ}$ Ano do Setor de Retina do Departamento de Oftalmologia do Hospital das Clínicas da Faculdade de Medicina da USP - São Paulo (SP) Brasil.

Endereço para correspondência: Otacílio de Oliveira Maia Júnior. Rua Adelmário Pinheiro, 10 - Salvador (BA) CEP 41900-540

E-mail: omaiausp@uol.com.br

Recebido para publicação em 24.11.2006

Última versão recebida em 26.06.2007

Aprovação em 30.07.2007

Nota Editorial: Depois de concluída a análise do artigo sob sigilo editorial e com a anuência do Dr. João Carlos Miranda Gonçalves sobre a divulgação de seu nome como revisor, agradecemos sua participação neste processo.

\section{INTRODUÇÃO}

O descolamento regmatogênico da retina (DRR) é caracterizado pela separação anatômica entre a camada neurossensorial e o epitélio pigmentado da retina (EPR), ficando esse espaço preenchido por líquido subretiniano, originário da cavidade vítrea, que migra através de uma descontinuidade da camada neurossensorial ${ }^{(1)}$. A reaplicação da retina no DRR é obtida através de bloqueio cirúrgico da ruptura retiniana, a retinopexia. As técnicas desta cirurgia incluem procedimentos epi-esclerais (introflexão escleral) ou vítreos (cirurgia pneumática e vitrectomia), sendo que as duas abordagens são freqüentemente associadas. O desenvolvimento de uma cicatriz entre a retina e a coróide por meio da crioterapia, diatermia ou fotocoagulação é essencial para bloquear as rupturas e manter a retina colada, utilizando-se freqüentemente um substituto vítreo temporário (ar, gás ou silicone) como adjuvante para obtenção desta finalidade ${ }^{(1-4)}$. 
A recuperação parcial da acuidade visual (AV), após uma cirurgia bem sucedida para DRR, pode ser devido a alterações como membranas epiretinianas, migração de pigmento, edema macular cistóide, buracos maculares, dobras retinianas e catarata. A presença de DRR por um longo período até a intervenção cirúrgica também pode cursar com baixa AV devido a alterações atróficas secundárias ${ }^{(5-6)}$. No entanto, mesmo na ausência destas complicações e nos casos com intervenção cirúrgica precoce, pode ocorrer uma baixa $\mathrm{AV}$ final após uma cirurgia anatomicamente bem sucedida ${ }^{(5)}$.

Com o advento da tomografia de coerência óptica, também conhecida pela sigla em inglês OCT, foi possível uma avaliação in vivo e não invasiva das camadas retinianas ${ }^{(7)}$. O OCT evidencia alterações estruturais pós-operatórias na região macular em cirurgias anatomicamente bem sucedidas, como a persistência de fluido subfoveal, não detectável tanto pela biomicroscopia quanto pela angiografia fluoresceínica $^{(6,8-9)}$. A influência destes achados têm sido associados à diminuição da $\mathrm{AV}$ e à metamorfopsia pósoperatória ${ }^{(6,8,10)}$, já que há melhora funcional após a reabsorção do fluido subfoveal ${ }^{(2,11-12)}$.

O objetivo deste trabalho é avaliar, por meio da acuidade visual e da tomografia de coerência óptica, a função e a estrutura da mácula de pacientes submetidos à cirurgia de descolamento regmatogênico da retina, anatomicamente bem sucedida.

\section{MÉTODOS}

Estudo prospectivo de pacientes do Departamento de Oftalmologia do Hospital das Clínicas da Faculdade de Medicina da Universidade de São Paulo (HC-FMUSP), submetidos à cirurgia de DRR sem intercorrências, anatomicamente bem sucedida, por meio de retinopexia pneumática ou introflexão escleral. Outros critérios de inclusão foram: indivíduos de ambos os sexos, qualquer idade, mácula descolada no préoperatório, sem tratamento retiniano prévio e sem outras doenças retinianas. Os critérios de exclusão foram: catarata, opacidades na córnea, complicações no pós-operatório como o descolamento recorrente, membranas epiretinianas, glaucoma, proliferação vítreo-retiniana, hemorragia vítrea e subretiniana, endoftalmite, edema macular cistóide, buraco macular ou dobras retinianas e perda de seguimento.

O tempo de DRR foi estimado pelo início da sintomatologia. A retinopexia foi associada à crioterapia. Nos casos de retinopexia pneumática, quando a sonda criogênica não atingia a ruptura, devido à altura elevada dos bolsões de retina, utilizou-se a fotocoagulação no pós-operatório, após a aplicação retiniana. A injeção do gás foi realizada com seringa e agulha de insulina a $4 \mathrm{~mm}$ do limbo na região superior do bulbo ocular, sendo utilizado $0,3 \mathrm{ml}$ de perfluor-propano $\left(\mathrm{C}_{3} \mathrm{~F}_{8}\right)$. A artéria central da retina e controle da pressão ocular foram avaliados durante uma hora após a injeção do gás. Para a técnica de introflexão escleral, utilizou-se pneu de silicone
287 e faixa de silicone 240 em todos os casos, sendo realizada drenagem externa do fluido sub-retiniano nos casos de bolsões elevados. A medicação padronizada para o pós-operatório, para ambas as técnicas, foi a associação de antibiótico e esteróides tópicos, atropina $1 \%$ tópica e antiinflamatório não esteróide via oral.

Durante o seguimento dos pacientes, foram estabelecidos dois exames oftalmológicos completos com ênfase na medida da AV com melhor correção (Tabela de Snellen), na biomicroscopia da mácula e no mapeamento da retina periférica. O primeiro exame ("follow-up" inicial) foi realizado por volta da $1^{\underline{q}}$ semana do pós-operatório, quando se fez o diagnóstico clínico de retina aplicada. O segundo exame ("follow-up" final) foi estabelecido em um período mínimo de três e máximo de seis meses, de acordo com a viabilidade da amostra. Os exames complementares realizados no seguimento foram a retinografia e OCT "Stratus version 4.0 software" (Carl Zeiss Meditec). As imagens obtidas pelo OCT foram realizadas no programa de medida da espessura da retina ("retinal thickness").

As variáveis quantitativas foram expressas em médias e desvios-padrão; para as qualitativas, calcularam-se freqüências absoluta e relativa. Para análise da AV no início e final do "follow-up", utilizou-se o teste não-paramétrico do sinal. Para o estudo de correlações entre duas variáveis, foi utilizado o coeficiente de correlação de Pearson ${ }^{(13)}$. O nível de significância utilizado para os testes foi de 5\%. O estudo foi aprovado pela Comissão de Ética para Análise de Projetos e Pesquisa da Diretoria Clínica do HC-FMUSP e todos os pacientes assinaram o termo de consentimento livre e esclarecido.

\section{RESULTADOS}

A amostra foi composta por 14 olhos (14 pacientes), 10 $(71,4 \%)$ submetidos à introflexão escleral e $4(28,6 \%)$, à retinopexia pneumática. A idade variou entre 24 e 59 anos (média de 39,3 $\pm 11,0$ anos), sendo $8(57,14 \%)$ pacientes do sexo masculino e $6(42,86 \%)$, do feminino. O tempo decorrido do DRR ao procedimento cirúrgico variou de 2 a 45 dias (19,8 $\pm 12,9$ dias). Todos os pacientes tiveram a retina aplicada na primeira semana do procedimento cirúrgico. A AV préoperatória variou de movimento de mãos a 0,1 e, no início do "follow-up" (diagnóstico de retina aplicada clinicamente), a acuidade variou de 0,05 a 1,0 $(0,49 \pm 0,3)$. O tempo de seguimento variou de 120 a 180 dias $(152,14 \pm 27,5$ dias $)$, momento em que a AV variou de 0,05 a $1,0(0,63 \pm 0,3)$ (Quadro 1).

Quanto às alterações no OCT, quatro olhos $(28,6 \%)$ apresentaram descolamento neurossensorial na região foveal e AV em média de 0,3 $\pm 0,25$ (Quadro 1- Pacientes 1, 3, 8 e 11). Todos os casos tiveram resolução espontânea do descolamento residual com melhora significante na acuidade, média de $0,75 \pm 0,20(\mathrm{p}=0,031)$; o tempo de resolução $(67,5 \pm 37,8$ dias $)$ não influenciou na acuidade final ( $\mathrm{p}=0,5546)$ (Figuras 1 e 2).

A análise de correlações evidenciou relação inversa entre 
a idade e AV final $(r=-0,64$ e $p=0,0127)$ e entre o tempo de descolamento e a AV final $(r=-0,54$ e $p=0,0447)$. Houve relação diretamente proporcional entre a AV inicial e final do "follow-up" (r=0,69 e p=0,0059).

\section{DISCUSS $\tilde{A} O$}

O tratamento cirúrgico do DRR apresenta sucesso anatômico superior a 90\% dos casos com a técnica de introflexão escleral e de 60 a $80 \%$, com a retinopexia pneumática. Entretanto, os resultados funcionais obtidos não correspondem aos anatômicos, obtendo-se visão melhor ou igual a $0,4 \mathrm{em}$ apenas $40 \%$ a $60 \%$ dos $\operatorname{casos}^{(14-16)}$. Apesar da maioria dos pacientes submetidos aos procedimentos cirúrgicos supracitados ter uma estabilidade visual no pós-operatório, a visão continua a melhorar mesmo após 5 anos da cirurgia ${ }^{(5)}$.

Recentemente, alguns autores não evidenciaram relação do tempo de DRR (até 30 dias) com a AV, mencionando que

Quadro 1. Características clínicas de pacientes submetidos à cirurgia de descolamento regmatogênico da retina, anatomicamente bem sucedida, por meio de retinopexia pneumática ou introflexão escleral

\begin{tabular}{|c|c|c|c|c|c|c|c|c|c|c|}
\hline Paciente & Idade & Sexo & Olho & $\begin{array}{l}\text { Tempo } \\
\text { DR }\end{array}$ & $\begin{array}{l}\text { AV } \\
\text { pré }\end{array}$ & Cirurgia & $\begin{array}{c}\text { AV } \\
\text { inicial }\end{array}$ & $\begin{array}{c}\text { Fluido } \\
\text { subfoveal OCT }\end{array}$ & $\begin{array}{l}\text { AV } \\
\text { final }\end{array}$ & $\begin{array}{c}\text { Tempo } \\
\text { "follow-up" }\end{array}$ \\
\hline 1 & 24 & $\mathrm{~F}$ & OD & $7 \mathrm{~d}$ & CD & RIE & 0,1 & presença & 0,7 & $120 \mathrm{~d}$ \\
\hline 2 & 58 & $\mathrm{M}$ & OD & $30 \mathrm{~d}$ & CD & RIE & 0,6 & não & 0,6 & $180 \mathrm{~d}$ \\
\hline 3 & 34 & $\mathrm{~F}$ & OE & $20 d$ & MM & RIE & 0,1 & presença & 0,5 & $180 \mathrm{~d}$ \\
\hline 4 & 45 & $\mathrm{M}$ & OD & $40 \mathrm{~d}$ & CD & RIE & 0,7 & não & 0,7 & $180 \mathrm{~d}$ \\
\hline 5 & 49 & $\mathrm{M}$ & OE & $15 d$ & CD & RIE & 0,3 & não & 0,3 & $150 \mathrm{~d}$ \\
\hline 6 & 59 & $\mathrm{~F}$ & OE & $45 \mathrm{~d}$ & MM & RIE & 0,05 & não & 0,05 & $180 d$ \\
\hline 7 & 37 & $\mathrm{M}$ & OD & $5 \mathrm{~d}$ & 0,05 & RIE & 0,8 & não & 0,8 & $120 \mathrm{~d}$ \\
\hline 8 & 42 & $\mathrm{M}$ & OD & $25 d$ & CD & RIE & 0,6 & presença & 0,8 & $150 \mathrm{~d}$ \\
\hline 9 & 47 & $\mathrm{~F}$ & OE & $18 d$ & CD & RIE & 0,2 & não & 0,3 & $180 \mathrm{~d}$ \\
\hline 10 & 29 & $\mathrm{M}$ & OD & $28 d$ & MM & RIE & 0,5 & não & 0,5 & $120 d$ \\
\hline 11 & 30 & $\mathrm{M}$ & $\mathrm{OE}$ & $20 d$ & 0,05 & $\mathrm{RP}$ & 0,4 & presença & 1,0 & $120 d$ \\
\hline 12 & 28 & $\mathrm{~F}$ & OD & $2 d$ & 0,1 & $\mathrm{RP}$ & 0,8 & não & 1,0 & $150 d$ \\
\hline 13 & 36 & $\mathrm{~F}$ & OD & $15 d$ & CD & $\mathrm{RP}$ & 0,7 & não & 0,6 & $180 d$ \\
\hline 14 & 32 & $\mathrm{M}$ & OD & $7 \mathrm{~d}$ & CD & $\mathrm{RP}$ & 1,0 & não & 1,0 & $120 \mathrm{~d}$ \\
\hline
\end{tabular}

$\mathrm{DR}=$ descolamento de retina; $\mathrm{AV}=$ acuidade visual (Tabela de Snellen): pré-operatória, inicial e final do "follow-up"; OCT= tomografia de coerência óptica; F= sexo feminino; $\mathrm{M}=$ sexo masculino, $\mathrm{OD}=$ olho direito; $\mathrm{OE}=$ olho esquerdo; $\mathrm{d}=$ dias; $\mathrm{CD}=$ conta dedos; $\mathrm{MM}=$ movimento de mãos; $\mathrm{RI}=$ = retinopexia com introflexão escleral; $\mathrm{RP}=$ retinopexia pneumática

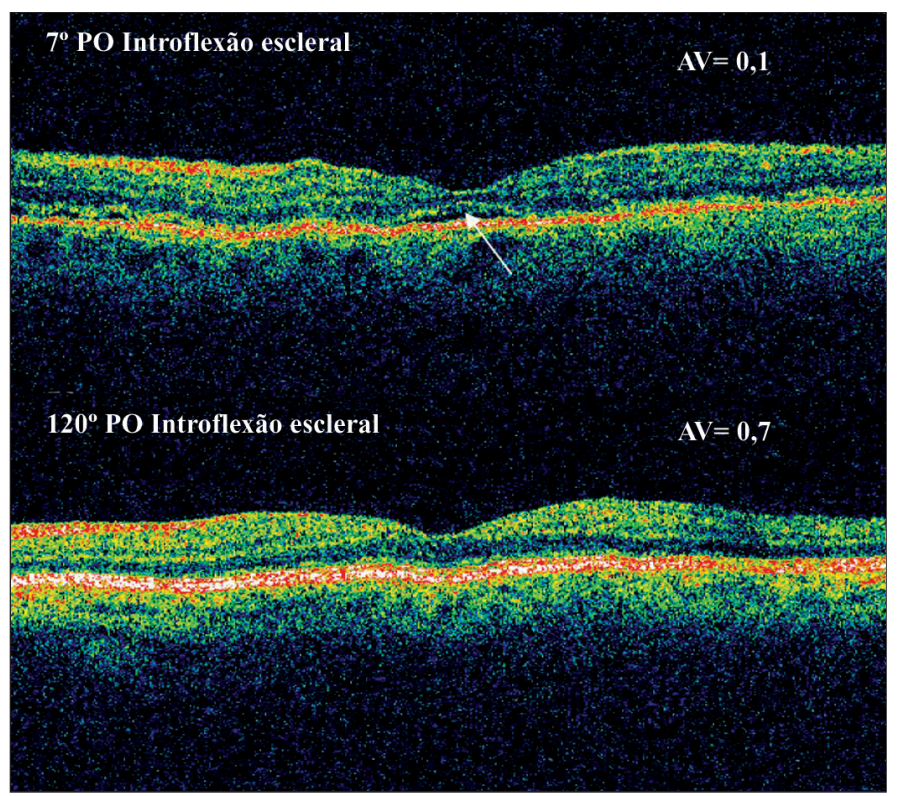

Figura 1 - Paciente 1, sexo feminino, 24 anos, 7 dias de DR com AV de conta dedos a 5 metros. Submetida a introflexão escleral, apresentou melhora da AV no 7을 $\mathrm{PO}$ OCT mostrando líquido residual na fóvea (seta). A imagem do Stratus OCT do seguimento mostrou ausência deste fluido no $120^{\circ} \mathrm{PO}$ com $\mathrm{AV}=0,7$

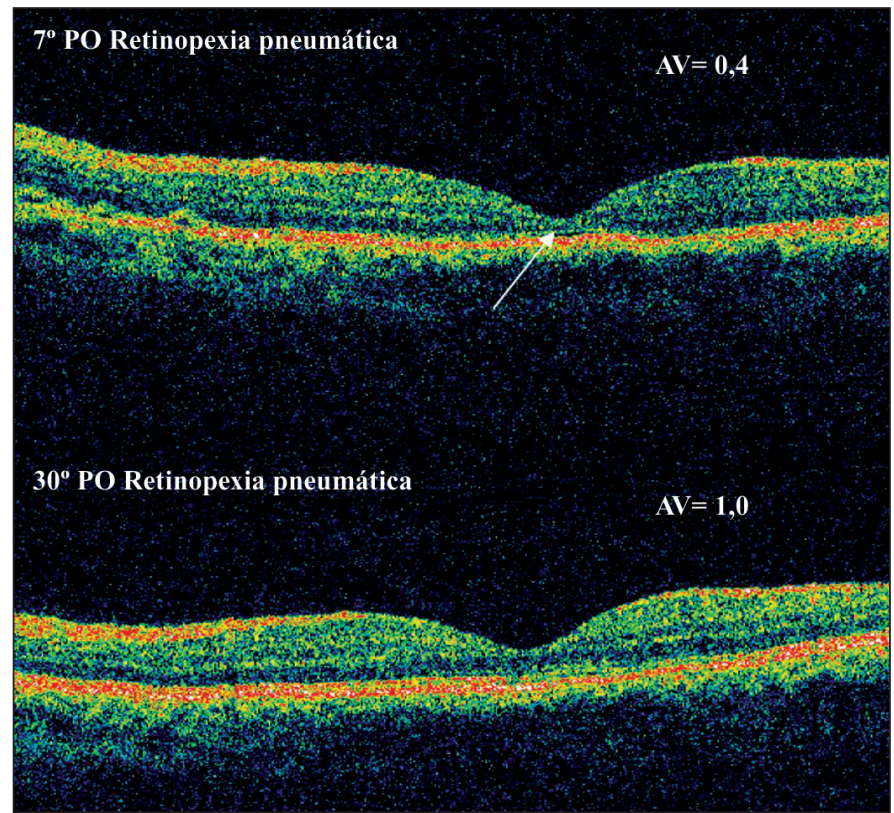

Figura 2 - Paciente 11, sexo masculino, 30 anos, 20 dias de DR com AV de 0,05 . Submetida a retinopexia pneumática, apresentou melhora da AV no $7^{\circ} \mathrm{PO}$ com OCT evidenciando descolamento foveal persistente (seta), o qual foi reabsorvido no $30^{\circ} \mathrm{PO} \operatorname{com} \mathrm{AV}=1,0$. 
acuidade pré-tratamento é um dos fatores mais importantes como prognóstico funcional ${ }^{(17)}$. Contudo, no presente trabalho foi constatada relação inversa entre o tempo de DRR (19,8 $\pm 12,9$ dias) e a AV final, resultados também demonstrados em estudos prévios ${ }^{(18-19)}$.

Com o advento do OCT, foi possível demonstrar a causa anatômica de alguns casos de insucesso funcional pós-operatório. A presença de líquido sub-retiniano na região macular, após cirurgia do DDR sem vitrectomia, pôde ser detectada pelo OCT em 47 a $81 \%$ dos pacientes após um mês de cirurgia $^{(4,6,9)}$. Alguns autores seguiram 15 pacientes submetidos à introflexão escleral, por meio do OCT, e observaram que em metade dos casos havia descolamento foveal ou parafoveal persistente com resolução espontânea num período de 12 meses $^{(8)}$. Usando o mesmo método de exame, Wolfensberger encontrou três categorias principais de imagens após a cirurgia de DRR: região foveal aplicada, descolamento difuso e plano da mácula e, mais comumente, um descolamento foveal circunscrito, com presença de fluido sub-retiniano residual $^{(11)}$. No presente estudo, a presença de líquido sub-retiniano subfoveal foi detectada pelo OCT em 4 olhos $(28,6 \%)$, incidência menor que a encontrada na literatura ${ }^{(8)}$, todos do tipo circunscrito.

Estudos prévios evidenciaram que em pacientes submetidos à vitrectomia, a incidência de líquido subfoveal persistente é menor, estando a região macular aplicada em 75 a $100 \%$ dos pacientes entre 4 e 6 semanas após a cirurgia ${ }^{(10-11)}$. A razão para esta diferença pós-cirúrgica na arquitetura foveal entre as duas técnicas permanece motivo de especulação, havendo evidências que o vítreo exerça papel importante nestes achados ${ }^{(11)}$.

Outro motivo de controvérsia é a origem do fluido subfoveal e a razão deste ficar confinado a uma pequena área no centro da mácula, como um descolamento subclínico. Especula-se que este líquido possa ser tanto fluido sub-retiniano residual quanto vazamento do EPR e/ou vasos da coróide adjacentes. Acredita-se, ainda, que possa ocorrer uma menor eficiência ou maior susceptibilidade a danos do mecanismo de bomba do EPR nesta região que no restante da retina ${ }^{(11,20)}$.

A influência desse descolamento foveal subclínico sobre a recuperação da $\mathrm{AV}$ ainda não está elucidada, podendo não influenciar na AV final ${ }^{(9,21)}$, mas sim aumentar incidência de metamorfopsia na vigência desta alteração macular ${ }^{(20)}$. Outros autores encontraram uma melhora da $\mathrm{AV}$ com a resolução do descolamento foveal e uma pior acuidade final nos casos em que este descolamento permaneceu por 12 meses após a cirurgia $^{(6)}$. O presente estudo, concordando com a literatura, revelou melhora na AV com a reabsorção do fluido subfoveal, entretanto, não mostrou correlação desta melhora com o tempo de resolução; possivelmente pelo número reduzido da amostra e por um tempo menor de resolução do quadro (máximo de 4 meses). Assim, tornam-se necessários estudos com um maior número de pacientes para melhor esclarecimento desta questão.
Outros fatores também podem estar relacionados a uma recuperação incompleta da AV após cirurgia vítreo-retiniana, entre eles estão o desalinhamento de fotorreceptores, perda do segmento externo de fotorreceptores, perda da linha de junção entre o segmento interno e externo dos fotorreceptores, mudanças degenerativas e hipertróficas do EPR, membrana epiretiniana e dobras retinianas ${ }^{(5-6,9,22)}$. Em estudo utilizando OCT de alta resolução, foi possível detectar rompimentos nos fotorreceptores na maioria dos pacientes com queixas visuais, após cirurgia por descolamento de retina. Este dano aos fotorreceptores pode explicar a recuperação incompleta da $\mathrm{AV}$, mesmo após a absorção do fluido subretiniano e em pacientes sem outras alterações estruturais detectáveis ${ }^{(22)}$. Alguns autores propuseram que a melhora lenta e gradual da acuidade após cirurgia se deve à recuperação de pigmentos dos cones, posteriormente a reaplicação retiniana ${ }^{(23)}$. Esta melhora, em geral, é mais sutil ${ }^{(5-6,9,22)}$, sendo que a melhora decorrente da reaplicação foveal, demonstrada no presente estudo, é mais evidente, o que está de acordo com outros autores ${ }^{(6)}$. Este fato tem importância clínica na orientação de um paciente que não está melhorando a AV após a cirurgia de DRR.

\section{CONCLUSÃO}

Em suma, a AV pós-operatória do descolamento de retina regmatogênico tem relação inversa com a idade e com tempo que a retina permaneceu descolada, ou seja, quanto mais jovem e mais precoce a intervenção cirúrgica, melhor AV final. Adicionalmente, há relação diretamente proporcional entre a AV na primeira semana do pós-operatório e a acuidade final, ou seja, quanto maior acuidade no início do pós-operatório, melhor acuidade final. Todos os casos de descolamento foveal evidenciados por meio da tomografia de coerência óptica no pós-operatório cursaram com reabsorção do líquido subfoveal e melhora da visão.

\section{ABSTRACT}

Purpose: To evaluate the structure and function of the macula following rhegmatogenous retinal detachment surgery. Methods: Prospective study of patients submitted to scleral buckle surgery or pneumatic retinopexy. The follow-up comprised a complete ophthalmologic exam and optical coherence tomography. Results: The sample was composed of 14 eyes (14 patients), $71.4 \%$ operated with the scleral buckle technique, and $28.6 \%$ with the pneumatic retinopexy. The age range was from 24 to 59 years (mean of 39.3 years). There was a negative correlation between the final visual acuity and age of patient $(r=-0.64, p=0.0127)$ and between final vision and duration of detachment $(\mathrm{r}=-0.54, \mathrm{p}=0.0447)$. There was a positive correlation between visual acuity at initial follow-up and that at the final follow-up $(\mathrm{r}=0.69, \mathrm{p}=0.0059)$. The optical coherence tomography of the initial follow-up showed subclinical foveal deta- 
chment in four eyes (28.6\% of cases); at the final follow-up the retina was applied by itself with improved vision in all eyes $(p=0.031)$, regardless the time span until reapplication $(\mathrm{p}=0.5546)$. Conclusion: The results show that the younger the patient and the earlier the surgical procedure, the better the final visual acuity. Furthermore, there is a positive correlation between initial and final postoperative vision and, all cases that presented foveal detachment on the optical coherence tomography at the initial follow-up had the retina flattened and the vision improved at the final examination.

Keywords: Retinal Detachment/diagnosis; Retinal Detachment / surgery; Macula lutea/physiopathology; Postoperative Complications; Tomography, optical coherence/methods; Visual acuity

\section{REFERÊNCIAS}

1. Farah ME. Descolamento e aderência da retina: contribuição à fisiopatologia e aos métodos de tratamento [tese]. São Paulo. Universidade Federal de São Paulo; 1998

2. Machemer R. The importance of fluid absorption, traction, intraocular currents and chorioretinal scars in the therapy of rhegmatogenous retinal detachments. XLI Edward Jackson memorial lecture. Am J Ophthalmol. 1984;98(6):681-93.

3. Goldhardt R, Corrêa ZM, Eichenberg MC, Marcon IM. Retinopexia com introflexão escleral em serviço de ensino de oftalmologia. Rev Bras Oftalmol. 2001;60(2):126-31.

4. Bonomo PP, Farah ME, Uno F. Retinopexia pneumática: estudo colaborativo de 51 casos. Arq Bras Oftalmol. 1996;59(1):23-6.

5. Ross WH, Kozy DW. Visual recovery in macula-off rhegmatogenous retinal detachments. Ophthalmology. 1998;105(11):2149-53. Comment in: Ophthalmology. 1999;106(9);1641-2

6. Wolfensberger TJ, Gonvers M. Optical coherence tomography in the evaluation of incomplete visual acuity recovery after macula-off retinal detachments. Graefes Arch Clin Exp Ophthalmol. 2002;240(2):85-9.

7. Huang d, Swanson EA, Lin CP, Schuman JS, Stinson WG, Chang W, et al. Optical coherence tomography. Science. 1991;254(5035):1178-81.
8. Hagimura N, Iida T, Suto K, Kishi S. Persistent foveal retinal detachment after successful rhegmatogenous retinal detachment surgery. Am J Ophthalmol. 2002;133(4):516-20

9. Lecleire-Collet A, Muraine M, Ménard JF, Brasseur G. Evaluation of macular changes before and after successful retinal detachment surgery using Stratusoptical coherence tomography. Am J Ophthalmol. 2006;142(1):176-9.

10. Benson SE, Schlottmann PG, Bunce C, Xing W, Charteris DG. Optical coherence tomography analysis of the macula after vitrectomy surgery for retinal detachment. Ophthalmology. 2006;113(7):1179-83.

11. Wolfensberger TJ. Foveal reattachment after macula-off retinal detachment occurs faster after vitrectomy than after buckle surgery. Ophthalmology. 2004;111(7):1340-3. Comment in: Ophthalmology. 2005;112(5):947; author reply $947-8$.

12. Theodossiadis GP, Georgalas IG, Emfietzoglou J, Kyriaki TE, Pantelia E, Gogas OS, et al. Optical coherence tomography findings in the macula after treatment of rhegmatogenous retinal detachments with spared macula preoperatively. Retina. 2003;23(1):69-75.

13. Rosner B. Fundamentals of biostatistics. $2^{\text {nd }}$ ed. Boston, PWS; 1986. 584 p.

14. The repair of rhegmatogenous retinal detachments. American Academy of Ophthalmology. Ophthalmology. 1990;97(11):1562-72.

15. Zaidi AA, Alvarado R, Irvine A. Pneumatic retinopexy: success rate and complications. Br J Ophthalmol. 2006;90(4):427-8.

16. Burton TC, Lambert RW Jr. A predictive model for visual recovery following retinal detachment surgery. Ophthalmology. 1978;85(6):619-25.

17. Salicone A, Smiddy WE, Venkatraman A, Feuer W. Visual recovery after scleral buckling procedure for retinal detachment. Ophthalmology. 2006; 113(10):1734-42.

18. Hassan TS, Sarrafizadeh R, Ruby AJ, Garretson BR, Kuczynski B, Williams GA. The effect of duration of macular detachment on results after the scleral buckle repair of primary, macula-off retinal detachments. Ophthalmology. 2002;109(1):146-52.

19. Abouzeid H, Wolfensberger TJ. Macular recovery after retinal detachment. Acta Ophthalmol Scand. 2006;84(5):597-605.

20. Wang Y, Li S, Zhu M, Chen SJ, Liu Y, Men XH, et al. Metamorphopsia after successful retinal detachment surgery: an optical coherence tomography study. Acta Ophthalmol Scand. 2005;83(2):168-71.

21. Baba T, Hirose A, Moriyama M, Mochizuki M. Tomographic image and visual recovery of acute macula-off rhegmatogenous retinal detachment. Graefes Arch Clin Exp Ophthalmol. 2004;242(7):576-81.

22. Schocket LS, Witkin AJ, Fujimoto JG, Ko TH, Schuman JS, Rogers AH, et al. Ultrahigh-resolution optical coherence tomography in patients with decreased visual acuity after retinal detachment repair. Ophthalmology. 2006; 113(4):666-72.

23. Liem AT, Keunen JE, van Meel GJ, van Norren D. Serial foveal densitometry and visual function after retinal detachment surgery with macular involvement. Ophthalmology. 1994;101(12):1945-52. 